\title{
Technique for chest compressions in adult CPR
}

\author{
Taufiek K Rajab ${ }^{1 *}$, Charles N Pozner ${ }^{2}$, Claudius Conrad ${ }^{3}$, Lawrence H Cohn ${ }^{4}$ and Jan D Schmitto ${ }^{5}$
}

\begin{abstract}
Chest compressions have saved the lives of countless patients in cardiac arrest as they generate a small but critical amount of blood flow to the heart and brain. This is achieved by direct cardiac massage as well as a thoracic pump mechanism. In order to optimize blood flow excellent chest compression technique is critical. Thus, the quality of the delivered chest compressions is a pivotal determinant of successful resuscitation. If a patient is found unresponsive without a definite pulse or normal breathing then the responder should assume that this patient is in cardiac arrest, activate the emergency response system and immediately start chest compressions. Contraindications to starting chest compressions include a valid Do Not Attempt Resuscitation Order. Optimal technique for adult chest compressions includes positioning the patient supine, and pushing hard and fast over the center of the chest with the outstretched arms perpendicular to the patient's chest. The rate should be at least 100 compressions per minute and any interruptions should be minimized to achieve a minimum of 60 actually delivered compressions per minute. Aggressive rotation of compressors prevents decline of chest compression quality due to fatigue. Chest compressions are terminated following return of spontaneous circulation. Unconscious patients with normal breathing are placed in the recovery position. If there is no return of spontaneous circulation, then the decision to terminate chest compressions is based on the clinical judgment that the patient's cardiac arrest is unresponsive to treatment. Finally, it is important that family and patients' loved ones who witness chest compressions be treated with consideration and sensitivity.
\end{abstract}

\section{Introduction}

Chest compressions have saved the lives of countless patients in cardiac arrest since they were first introduced in 1960 [1]. Cardiac arrest is treated with cardiopulmonary resuscitation $(\mathrm{CPR})$ and chest compressions are a basic component of CPR. The quality of the delivered chest compressions is a pivotal determinant of successful resuscitation [2]. In spite of this, studies show that the quality of chest compressions, even if delivered by healthcare professionals, is often suboptimal [2]. Therefore it is important that providers carefully familiarize themselves with this technique.

\section{Indications}

Chest compressions are generally indicated for all patients in cardiac arrest. Unlike other medical interventions, chest compressions can be initiated by any healthcare provider without a physician's order. This is based on implied patient consent for emergency treatment [3].

\footnotetext{
* Correspondence: trajab@rics.bwh.harvard.edu

'Department of Surgery, Brigham and Women's Hospital, Harvard Medical School, Boston, MA, USA

Full list of author information is available at the end of the article
}

If a patient is found unresponsive without a definite pulse or normal breathing then the responder should assume that this patient is in cardiac arrest, activate the emergency response system and immediately start chest compressions [4]. The risk of serious injury from chest compressions to patients who are not in cardiac arrest is negligible [5], while any delay in starting chest compressions has grave implications for outcome. Due to the importance of starting chest compressions early, pulse and breathing checks were de-emphasized in the most recent CPR guidelines [4]. Thus, healthcare providers should take no longer than 10 seconds to check for a pulse. The carotid or femoral pulses are preferred locations for pulse checks since peripheral arteries can be unreliable.

\section{Contraindications}

In certain circumstances it is inappropriate to initiate chest compressions. A valid Do Not Resuscitate (DNR) order that prohibits chest compressions is an absolute contra-indication. DNR orders are considered by the attending physician on the basis of patient autonomy and treatment futility.
C Biomed Central

C 2011 Rajab et al; licensee BioMed Central Ltd. This is an Open Access article distributed under the terms of the Creative Commons Attribution License (http://creativecommons.org/licenses/by/2.0), which permits unrestricted use, distribution, and reproduction in any medium, provided the original work is properly cited. 
The principle of patient autonomy dictates that competent patients have a right to refuse medical treatment [6]. Therefore a DNR order should be documented if patients do not wish to be treated with chest compressions. For patients with impaired decision-making, previous preferences should be taken into account when making decisions regarding DNR.

The principle of treatment futility dictates that healthcare providers are not obliged to provide treatment if this would be futile [6]. Therefore a DNR order should be documented if chest compressions would be unlikely to confer a survival benefit or acceptable quality of life. However, few criteria can reliably predict the futility of starting chest compressions.

If there is any uncertainty regarding DNR status then chest compressions should be started immediately while the uncertainties are addressed. Compressions may subsequently be terminated as soon as a valid DNR order is produced.

Of note, patients with implantable left ventricular assist devices [7-9] or patients with total artificial hearts or biventricular assist devices [10] who suffer cardiac arrest from device failure should be resuscitated using a backup pump (e.g. ECMO [11,12]) if this is available rather than with chest compressions.

\section{The Physiology of Chest Compressions}

Chest compressions generate a small but critical amount of blood flow to the heart and brain. This significantly improves the chances of successful resuscitation [13]. However, the precise mechanism of blood flow during chest compressions has been controversial since the 1960s. The two main hypotheses are the external cardiac massage model and the thoracic pump model.

The external cardiac massage model suggests that chest compressions directly compress the heart between the depressed sternum and the thoracic spine [1]. This ejects blood into the systemic and pulmonary circulations while backward flow during decompression is limited by the cardiac valves. The external cardiac massage model is supported by radiographic evidence of direct compression of cardiac structures during chest compressions [14].

The thoracic pump model suggests that chest compressions intermittently increase global intra-thoracic pressure, with equivalent pressures exerted on vena cava, the heart and the aorta [9]. Thus blood is ejected retrograde from the intra-thoracic venous vasculature as well as antegrade from the intra-thoracic arterial vasculature and both arterial as well as venous pressures rise concomitantly. Therefore the presence of an arterial pulse in itself is not a reliable indicator of blood flow. This principle is illustrated by the fact that a ligated artery will continue to pulsate even in the absence of blood flow. However, the compliance of venous capacitance vessels is greater than the compliance of arterial resistance vessels. Therefore a pressure differential between the extra-thoracic arterial and venous sides of the vascular tree is formed. This pressure differential is but a fraction of the arterial pulse pressure, yet it is sufficient to drive some blood flow. The thoracic pump model is supported by arterial and venous pressure tracings demonstrating simultaneous peaks in venous and arterial pressures during chest compressions [15].

In toto, the available evidence suggests that both cardiac massage and the thoracic pump contribute to blood flow during chest compressions. Yet even excellent chest compressions can only generate a fraction of baseline blood flow [16]. Therefore the time during chest compressions contributes to the ongoing ischemic insult to the patient's heart and brain.

The brain is the organ most susceptible to decreased blood flow and suffers irreversible damage within 5 minutes of absent perfusion. The myocardium is the second most susceptible organ, with ROSC directly related to coronary perfusion pressures [17]. Therefore successful resuscitation with neurologically intact survival and ROSC critically depends on maintaining blood flow to the heart and brain via chest compressions.

\section{Technique for Chest Compression}

Chest compressions consist of forceful and fast oscillations of the lower half of the sternum [1]. The technique of delivering chest compressions is highly standardized and based on international consensus that is updated in 5 -year intervals $[4,13,18]$.

\section{Patient Positioning}

The patient in cardiac arrest should be placed in supine position with the rescuer standing beside the patient's bed or kneeling beside the patient's chest [18]. Adjustment of the bed height or standing on a stool allows leveraging the body weight above the waist for mechanical advantage. For optimal transfer of energy during chest compressions the patient should be positioned on a firm surface such as a backboard early in resuscitation efforts. This decreases wasting of compressive force by compression of the soft hospital bed. While re-positioning the patient, interruptions of chest compressions should be minimized and care should be taken to avoid dislodging any lines or tubes [13].

\section{Hand Position and Posture}

Place the dominant hand over the center of the patient's chest [19]. This position corresponds to the lower half of the sternum. The heel of the hand is positioned in the midline and aligned with the long axis of the sternum. This focuses the compressive force on the sternum 
and decreases the chance of rib fractures. Next, place the non-dominant hand on top of the first hand so that both hands are overlapped and parallel. The fingers should be elevated off the patient's ribs to minimize compressive force over the ribs. Also avoid compressive force over the xiphisternum or the upper abdomen to minimize iatrogenic injury.

The previously taught method of first identifying anatomical landmarks and then positioning the hands two centimeters above the xiphoid-sternal notch was found to prolong interruptions of chest compressions without an increase in accuracy [20]. Similarly, the use of the internipple line as a landmark for hand placement was found to be unreliable [21]. Therefore these techniques are no longer part of the international consensus guidelines $[4,13,18]$.

For maximum mechanical advantage keep your arms straight and elbows fully extended. Position your shoulders vertically above the patient's sternum. If the compressive force is not perpendicular to the patient's sternum then the patient will roll and part of the compressive force will be lost.

\section{Compression Rate and Interruptions}

The blood flow generated by chest compressions is a function of the number of chest compressions delivered per minute and the effectiveness of each chest compression. The number of compressions delivered per minute is clearly related to survival [22]. This depends on the rate of compressions and the duration of any interruptions. Chest compressions should be delivered at a rate of at least 100 compressions per minute [4] since chest compression rates below $80 / \mathrm{min}$ are associated with decreased ROSC [2]. Any interruptions of chest compressions should be minimized. Legitimate reasons to interrupt chest compressions include the delivery of non-invasive rescue breaths, the need to assess rhythm or ROSC, and defibrillation [18]. Hold compressions when non-invasive rescue breaths are delivered [18]. Once an advanced airway is established there is no need to hold compressions for further breaths. High-quality compressions must also continue while defibrillation pads are applied and the defibrillator is prepared [13]. Aim to minimize interruption of chest compressions during the changeover of rescuers. Including all interruptions the patient should receive at least 60 compressions per minute [13].

\section{Compression Depth, Recoil and Duty Cycle}

Compression depth should be at least $5 \mathrm{~cm}$, since sternal depression of $5 \mathrm{~cm}$ and over results in a higher ROSC [18]. No upper limit for compression depth has been established in human studies but experts recommend that sternal depression should not exceed $6 \mathrm{~cm}$ [13].
After each compression, allow the chest to recoil completely. Incomplete recoil results in worse hemodynamics, including decreased cardiac perfusion, cerebral perfusion and cardiac output [23]. Complete recoil is achieved by releasing all pressure from the chest and not leaning on the chest during the relaxation phase of the chest compressions [13]. However, avoid lifting the hands off the patient's chest, since this was associated with a reduction in compression depth [24].

The duration of the compression phase as a proportion of the total cycle is termed duty cycle. Although duty cycles ranging between $20 \%$ and $50 \%$ can result in adequate cardiac and cerebral perfusion [25], a duty cycle of $50 \%$ is recommended because it is easy to achieve with practice [4]. Thus the duration of the compression phase should be equivalent to the duration of the decompression phase. If the patient has hemodynamic monitoring via an arterial line then compression rate, compression depth and recoil can be optimized for the individual patient on the basis of this data.

\section{Rotating Rescuers}

The quality of chest compressions deteriorates over time due to fatigue [26]. Therefore the compressor should be rotated every two minutes [13]. Rotating compressors more frequently than this may have detrimental effects due to interruptions of chest compressions from the practicalities of the changeover [27]. Consider rotating compressors during any intervention associated with appropriate interruptions of chest compressions, for example when defibrillating. Every effort should be made to accomplish the switch in less than five seconds. For this purpose it may be helpful for the compressor performing chest compressions to count out loud [13]. If the rotating compressors can be positioned on either side of the patient, one compressor can be ready and waiting to relieve the working compressor in an instant [4].

\section{Termination of Efforts}

Chest compressions are terminated following ROSC and unconscious patients with normal breathing are placed in the recovery position [28]. If there is no ROSC, then the decision to terminate efforts is based on the clinical judgment that the patient's arrest is unresponsive to treatment. This decision should be made by the physician leading the emergency response team after consultation with the members of the team. The factors that are considered include the time to initiate chest compressions, duration of chest compressions, initial arrest rhythm, age, comorbidities and any reversible causes of cardiac arrest such as drug overdose [3]. The duration of cardiac arrest is the most important prognostic factor [29]. In general, chest compressions should be 
continued at least as long as VF persists. Prolonged chest compressions are less likely to succeed if there is no ROSC within half an hour. However, case reports with exceptional ROSC are well documented and each decision to terminate efforts should be made individually. Any family members and patients' loved ones who witness chest compressions should be treated with consideration and sensitivity.

\section{Complications}

Life-threatening complications of chest compressions are extremely rare [24]. Such complications occur less frequently than $1 \%$ [30-35]. If hypotension is noted following ROSC then cardiogenic shock and abdominal injury are the most important complications of chest compressions that should be considered [31]. Rib fractures are the most frequent complication, with an incidence of $1 / 3$ at autopsy [30]. However, rib fractures were noted in only $2 \%$ of non-arrest patients who received chest compressions from a bystander [5]. Following successful ROSC all patients should be re-evaluated for resuscitation-related injuries [28].

\section{Summary}

High quality chest compressions are proven to save lives. If an unresponsive patient has no definite pulse or is not breathing normally then the responder should assume that this patient is in cardiac arrest, activate the emergency response system and immediately start chest compressions. Push hard and fast over the center of the chest. Minimize interruptions of chest compressions and aggressively rotate compressors. Following successful ROSC place the patient in the recovery position and reevaluate for resuscitation-related injuries. If there is no reasonable chance for ROSC then the decision to terminate efforts should be made by the leader of the emergency response team. Any family members witnessing chest compressions should be treated with sensitivity and respect.

\footnotetext{
Author details

'Department of Surgery, Brigham and Women's Hospital, Harvard Medical School, Boston, MA, USA. ²Department of Emergency Medicine, Brigham and Women's Hospital, Harvard Medical School, Boston, MA, USA. ${ }^{3}$ Department of Surgery, Massachusetts General Hospital, Harvard Medical School, Boston, MA, USA. ${ }^{4}$ Division of Cardiac Surgery, Brigham and Women's Hospital, Harvard Medical School, Boston, MA, USA. ${ }^{5}$ Department of Cardiac, Thoracic, Transplantation and Vascular Surgery, Hannover Medical School, Hannover, Germany.
}

\section{Authors' contributions}

TKR conceived the study, performed literature search and drafted the manuscript. CNP participated in the design of the study, was involved in drafting the manuscript and is responsible revising it critically. CC was involved in drafting the manuscript and critically revised it. LHC participated in the design of the study, was involved in drafting the manuscript and is responsible revising it critically. JDS conceived of the study, was involved in drafting the manuscript and participated in its design and coordination. All authors read and approved the final manuscript.

\section{Competing interests}

The authors declare that they have no competing interests.

Received: 4 October 2011 Accepted: 10 December 2011

Published: 10 December 2011

\section{References}

1. Kouwenhoven W, Jude J, Knickerbocker G: Closed-chest cardiac massage. JAMA 1960, 173:1064-7.

2. Abella BS, et al: Chest compression rates during cardiopulmonary resuscitation are suboptimal: a prospective study during in-hospital cardiac arrest. Circulation 2005, 111(4):428-34

3. Morrison LJ, et al: Part 3: ethics: 2010 American Heart Association Guidelines for Cardiopulmonary Resuscitation and Emergency Cardiovascular Care. Circulation 2010, 122(18 Suppl 3):S665-75.

4. Berg RA, et al: Part 5: adult basic life support: 2010 American Heart Association Guidelines for Cardiopulmonary Resuscitation and Emergency Cardiovascular Care. Circulation 2010, 122(18 Suppl 3): S685-705.

5. White $L$, et al: Dispatcher-assisted cardiopulmonary resuscitation: risks for patients not in cardiac arrest. Circulation 2010, 121(1):91-7.

6. Lippert FK, et al: European Resuscitation Council Guidelines for Resuscitation 2010 Section 10. The ethics of resuscitation and end-of-life decisions. Resuscitation 2010, 81(10):1445-51.

7. Mokashi SA, Schmitto JD, Lee LS, Rawn JD, Bolman RM, Shekar PS, Couper GS, Chen FY: Ventricular assist device in patients with prosthetic heart valves. Artif Organs 2010, 34(11):1030-4.

8. Schmitto JD, Molitoris U, Haverich A, Strueber M: Implantation of a centrifugal pump as a left ventricular assist device through a novel, minimized approach: Upper hemisternotomy combined with anterolateral thoracotomy. J Thorac Cardiovasc Surg 2011.

9. Mokashi SA, Guan J, Wang D, Tchantchaleishvili V, Brigham M, Lipsitz S, Lee LS, Schmitto JD, Bolman RM, Khademhosseini A, Liao R, Chen FY: Preventing cardiac remodeling: the combination of cell-based therapy and cardiac support therapy preserves left ventricular function in rodent model of myocardial ischemia. J Thorac Cardiovasc Surg 2010, 140(6):1374-80.

10. Strueber M, Schmitto JD, Kutschka I, Haverich A: Placement of two implantable centrifugal pumps to serve as a total artificial heart after cardiectomy. J Thorac Cardiovasc Surg 2011.

11. Coskun KO, Popov AF, Schmitto JD, Hinz J, Kriebel T, Schoendube FA Ruschewski W, Tirilomis T: Extracorporeal circulation for rewarming in drowning and near-drowning pediatric patients. Artif Organs 2010, 34(11):1026-30.

12. Coskun KO, Coskun ST, Popov AF, Hinz J, El-Arousy M, Schmitto JD, Kececioglu D, Koerfer R: Extracorporeal life support in pediatric cardiac dysfunction. J Cardiothorac Surg 2010, 5:112.

13. Koster RW, et al: European Resuscitation Council Guidelines for Resuscitation 2010 Section 2. Adult basic life support and use of automated external defibrillators. Resuscitation 2010, 81(10):1277-92.

14. Hwang SO, et al: Compression of the left ventricular outflow tract during cardiopulmonary resuscitation. Acad Emerg Med 2009, 16(10):928-33.

15. Weale FE, Rothwell-Jackson RL: The efficiency of cardiac massage. Lancet 1962, 1(7237):990-2.

16. Delguercio $L R$, et al: Comparison of blood flow during external and internal cardiac massage in man. Circulation 1965, 31(SUPPL 1):171-80.

17. Paradis N, et al: Coronary perfusion pressure and the return of spontaneous circulation in human cardiopulmonary resuscitation. JAMA 1990, 263(8):1106-13

18. Sayre MR, et al: Part 5: Adult basic life support: 2010 International Consensus on Cardiopulmonary Resuscitation and Emergency Cardiovascular Care Science With Treatment Recommendations. Circulation 2010, 122(16 Suppl 2):S298-324.

19. Kundra P, Dey S, Ravishankar M: Role of dominant hand position during external cardiac compression. Br J Anaesth 2000, 84(4):491-3.

20. Handley AJ: Teaching hand placement for chest compression-a simpler technique. Resuscitation 2002, 53(1):29-36. 
21. Shin J, Rhee JE, Kim K: Is the inter-nipple line the correct hand position for effective chest compression in adult cardiopulmonary resuscitation? Resuscitation 2007, 75(2):305-10.

22. Christenson J, et al: Chest compression fraction determines survival in patients with out-of-hospital ventricular fibrillation. Circulation 2009, 120(13):1241-7.

23. Yannopoulos D, et al: Effects of incomplete chest wall decompression during cardiopulmonary resuscitation on coronary and cerebral perfusion pressures in a porcine model of cardiac arrest. Resuscitation 2005, 64(3):363-72.

24. Aufderheide TP, et al: Incomplete chest wall decompression: a clinical evaluation of CPR performance by EMS personnel and assessment of alternative manual chest compression-decompression techniques. Resuscitation 2005, 64(3):353-62.

25. Handley AJ, Handley JA: The relationship between rate of chest compression and compression:relaxation ratio. Resuscitation 1995, 30(3):237-41.

26. Hightower $D$, et al: Decay in quality of closed-chest compressions over time. Ann Emerg Med 1995, 26(3):300-3.

27. Manders S, Geijsel FE: Alternating providers during continuous chest compressions for cardiac arrest: every minute or every two minutes? Resuscitation 2009, 80(9):1015-8.

28. Resuscitation IL 2005 International Consensus on Cardiopulmonary Resuscitation and Emergency Cardiovascular Care Science with Treatment Recommendations. Part 2: Adult basic life support. Resuscitation 2005, 67(2-3):187-201.

29. Schultz SC, et al: Predicting in-hospital mortality during cardiopulmonary resuscitation. Resuscitation 1996, 33(1):13-7.

30. Krischer JP, et al: Complications of cardiac resuscitation. Chest 1987 92(2):287-91.

31. Atcheson SG, Fred HL: Letter: Complications of cardiac resuscitation. Am Heart J 1975, 89(2):263-5.

32. Schmitto JD, Rajab TK, Cohn LH: Prevalence and variability of internal mammary graft use in contemporary multivessel coronary artery bypass graft. Curr Opin Cardiol 2010, 25(6):609-12.

33. Schmitto JD, Mokashi SA, Cohn LH: Past, present, and future of minimally invasive mitral valve surgery. J Heart Valve Dis 2011, 20(5):493-8.

34. Schmitto JD, Mohr FW, Cohn LH: Minimally invasive aortic valve replacement: how does this perform in high-risk patients? Curr Opin Cardiol 2011, 26(2):118-22.

35. Schmitto JD, Mokashi SA, Cohn LH: Minimally-invasive valve surgery. J Am Coll Cardiol 2010, 56(6):455-62.

doi:10.1186/1749-7922-6-41

Cite this article as: Rajab et al:: Technique for chest compressions in adult CPR. World Journal of Emergency Surgery 2011 6:41.

\section{Submit your next manuscript to BioMed Central and take full advantage of:}

- Convenient online submission

- Thorough peer review

- No space constraints or color figure charges

- Immediate publication on acceptance

- Inclusion in PubMed, CAS, Scopus and Google Scholar

- Research which is freely available for redistribution

Submit your manuscript at www.biomedcentral.com/submit
Biomed Central 